\title{
Establishment of Opioid-Induced Rewarding Effects Under Oxaliplatin- and Paclitaxel-Induced Neuropathy in Rats
}

\author{
Tomohisa Mori ${ }^{1, \dagger}$, Tomoe Kanbara ${ }^{1,2, \dagger}$, Masato Harumiya ${ }^{1}$, Yoshiyuki Iwase ${ }^{1}$, Aki Masumoto ${ }^{1}$, \\ Sachiko Komiya ${ }^{1}$, Atsushi Nakamura ${ }^{1,2}$, Masahiro Shibasaki ${ }^{1}$, Toshiyuki Kanemasa ${ }^{2}$, Gaku Sakaguchi ${ }^{2}$, \\ and Tsutomu Suzuki ${ }^{1, *}$ \\ ${ }^{1}$ Department of Toxicology, Hoshi University School of Pharmacy and Pharmaceutical Sciences, \\ 2-4-41 Ebara, Shinagawa-ku, Tokyo 142-8501, Japan \\ ${ }^{2}$ Pain \& Neurology, Discovery Research Laboratories, Shionogi \& Co., Ltd., \\ 1-1, 3-chome, Futaba-cho, Toyonaka, Osaka 561-0825, Japan
}

Received May 29, 2014; Accepted July 3, 2014

\begin{abstract}
The rewarding effects of $\mu$-receptor agonists can be suppressed under several pain conditions. We recently showed that clinically used $\mu$-receptor agonists possess efficacies for relieving the neuropathic pain induced by chemotherapeutic drug in rats; however, it is possible that the use of $\mu$-receptor agonists may trigger the rewarding effects even under chemotherapeutic drug-induced neuropathic pain. Nevertheless, no information is available regarding whether $\mu$ receptor agonists produce psychological dependence under chemotherapeutic drug-induced neuropathic pain. Therefore, we examined the effects of neuropathy induced by chemotherapeutic drugs on the rewarding effects of morphine, oxycodone, and fentanyl in rats. Repeated treatment with oxaliplatin or paclitaxel produced neuropathy as measured by the von Frey test. Rewarding effects produced by antinociceptive doses of $\mu$-receptor agonists were not suppressed under oxaliplatin- or paclitaxel-induced neuropathy. Furthermore, the morphine-induced increase in the release of dopamine from the nucleus accumbens, which is a critical step in the rewarding effects of $\mu$-receptor agonists, was not altered in paclitaxel-treated rats. These results suggest that the rewarding effects of $\mu$-receptor agonists can still be established under oxaliplatin- or paclitaxelinduced neuropathic pain. Therefore, patients should be carefully monitored for psychological dependence on $\mu$-receptor agonists when they are used to control chemotherapeutic drug-induced neuropathic pain.
\end{abstract}

Keywords: rewarding effect, oxaliplatin, paclitaxel, neuropathic pain, $\mu$-opioid receptor agonist

\section{Introduction}

Although $\mu$-opioid receptor agonists such as morphine, oxycodone, and fentanyl have prominent antinociceptive effects, they also have adverse effects (e.g., emesis, constipation, drowsiness, and psychological dependence). Psychological dependence on opioids is a serious problem worldwide, and one of the triggers for inducing such opioid dependence is the inappropriate use or

\footnotetext{
†These authors contributed equally to this work.

*Corresponding author. suzuki@hoshi.ac.jp

Published online in J-STAGE on August 20, 2014

doi: 10.1254/jphs.14134FP
}

overdose of prescribed $\mu$-opioid receptor agonists (1) It is widely accepted that psychological events such as the reinforcing effects induced by $\mu$-opioid receptor agonists can be mimicked in animals as rewarding effects $(2-4)$, and activation of the mesolimbic dopaminergic system, which projects from the ventral tegmental area (VTA) to the nucleus accumbens (NAc), plays an important role in the rewarding effects of $\mu$-opioid receptor agonists $(5-8)$.

Recently, it has been shown that, as long as $\mu$-opioid receptor agonists are used appropriately to control pain, psychological dependence does not occur in clinical situations $(9,10)$. We have previously demonstrated that the rewarding effects of morphine are suppressed 
under inflammatory (carrageenan- and formalin-induced pain) and neuropathic pain (partial sciatic nerve ligation)like states in rodents as measured by the conditioned place preference paradigm, and this is accompanied by inactivation of the mesolimbic dopaminergic system $(11-13)$. The mechanisms of action for suppression of the activation of the mesolimbic dopaminergic system under inflammatory or neuropathic pain by morphine are mediated though totally different pathways; inflammatory pain upregulates the function of the dynorphinergic system in the NAc (11), whereas neuropathic pain downregulates $\mu$-opioid receptor function in the VTA (14).

Chemotherapeutics, such as oxaliplatin and paclitaxel, often cause adverse side effects, like diarrhea, emesis, muscle pain, joint pain, and peripheral neuropathy (15). Among these adverse events, peripheral neuropathy is one of the most serious adverse effects during long-term cancer therapy, and uncontrollable pain management limits the use of chemotherapy drugs. Recently, clinical studies have suggested that oxycodone, a $\mu$-opioid receptor agonist, is effective for reducing oxaliplatin- or paclitaxel-induced neuropathic pain $(16,17)$. In addition, morphine and oxycodone, but not fentanyl, relieved oxaliplatin-induced neuropathic pain in rats (submitted manuscript), suggesting that some $\mu$-opioid receptor agonists have beneficial effects on chemotherapyinduced peripheral neuropathy.

To the best our knowledge, no information is available regarding whether psychological dependence on $\mu$-opioid receptor agonists would be a major concern under chronic neuropathy conditions induced by chemotherapeutics in a clinical setting. Although chemotherapeutic drug-induced neuropathy is generally classified as neuropathic pain, its symptoms seem to be different from those of general neuropathic pain $(18-20)$. Chemotherapeutic agents induce a distinct characteristic neuropathy compared with neuropathies due to other causes, and chemotherapeutic drug-induced neuropathy may affect $\mu$-opioid receptor agonist-induced activation of the mesolimbic dopaminergic system and regulate the establishment of the rewarding effects of $\mu$-opioid receptor agonists. Therefore, we examined the effects of the neuropathy induced by chemotherapeutic drugs (oxaliplatin and paclitaxel) on the establishment of the rewarding effects of $\mu$-opioid receptor agonists in rats.

\section{Materials and Methods}

\section{Animals}

Male Sprague Dawley rats (160 to 300 g) (Charles River Laboratories Japan, Kanagawa) were used. Food and water were available ad libitum for rats in their home cages. Animals were housed in a room maintained at $22^{\circ} \mathrm{C} \pm 1{ }^{\circ} \mathrm{C}$ with a 12 -h light-dark cycle (light on 8:00 a.m. to 8:00 p.m.). The present study was conducted in accordance with animal protocols approved by the Institutional Animal Care and Use Committee of Shionogi Research Laboratories (Osaka) and the Guiding Principles for the Care and Use of Laboratory Animals at Hoshi University adopted by the Committee on Animal Research of Hoshi University. Every effort was made to minimize the numbers and any suffering of animals used in the following experiments.

\section{Chemotherapy-induced peripheral neuropathy}

Oxaliplatin-induced peripheral neuropathy was initiated as described previously (submitted manuscript). Briefly, oxaliplatin $(2 \mathrm{mg} / \mathrm{kg})$ or vehicle was administered intraperitoneally (i.p.) twice a week for 4 weeks (days 1, 2, 8, 9, 15, 16, 22, and 23). Paclitaxel-induced mechanical allodynia, which is based on a previous report (21), was induced by multiple i.p. injections of paclitaxel $(1 \mathrm{mg} / \mathrm{kg})$ or vehicle on days $1,2,3,4,5,8,9,10,11$, and 12. The latency of paw withdrawal was assessed before each injection of paclitaxel on days $1,2,3,5,8$, 11,16 , and 17. The conditioned place preference test and in vivo microdialysis studies were initiated after chemotherapy-induced peripheral neuropathy was confirmed.

\section{von Frey hair test for mechanical allodynia}

Mechanical allodynia was assessed by the von Frey monofilament test as described previously (submitted manuscript). Briefly, von Frey filaments $(0.6,1,1.4,2$, $4,6,8,10,15$, and $26 \mathrm{~g}$ ) were applied to each hind paw for a maximum period of $4 \mathrm{~s}$, and the withdrawal response was observed. The 8-g stimulus was applied first, and the weakest stimulation that caused a positive response was determined as the threshold value using the up-down method. A change in the threshold from $8-15 \mathrm{~g}$ before the first injection of paclitaxel or oxaliplatin to $<4 \mathrm{~g}$ at $16-18$ days after the first administration of paclitaxel or $36-38$ days after the first administration of oxaliplatin was used in the experiments. To compare the analgesic effects of the three opioids in the paclitaxel model, the antinociceptive effect was expressed as a percentage of the maximal possible effect (\%MPE) and calculated as $(\mathrm{T} 1-\mathrm{T} 0) \times 100 /(\mathrm{T} 2-\mathrm{T} 0)$, where $\mathrm{T} 0$ is the mean of the paw-withdrawal threshold in the paclitaxel-saline group, $\mathrm{T} 1$ is the paw-withdrawal threshold of each animal treated with drug, and T2 is the mean of the paw-withdrawal threshold in the sham-saline group.

\section{Place conditioning}

Place-conditioning studies were conducted using a shuttle box $(30 \times 60 \times 30 \mathrm{~cm}: \mathrm{w} \times 1 \times \mathrm{h})$ that was made of an acrylic resin board and divided into two equal-sized 
compartments (22). One compartment was white with a textured floor and the other was black with a smooth floor to create equally inviting compartments. The place-conditioning schedule consisted of 3 phases (preconditioning test, conditioning, and post-conditioning test). The pre-conditioning test was performed as follows: the partition separating the two compartments was raised to $7-\mathrm{cm}$ above the floor, a neutral platform was inserted along the seam separating the compartments, and rats that had not been treated with either drugs or saline were then placed on the platform. The time spent in each compartment during a $900-\mathrm{s}$ session was recorded automatically using an infrared beam sensor ( $\mathrm{KN}-80$; Natsume Seisakusyo Co., Ltd., Tokyo). After paclitaxelor oxaliplatin-induced allodynia was observed, conditioning sessions (three for drug: three for saline) were started the day after the pre-conditioning test and conducted once daily for 6 days. Immediately after the injection of drug (s.c.), these animals were placed in the compartment opposite that in which they had spent the most time in the pre-conditioning test for $1 \mathrm{~h}$. On alternate days, these animals were treated with saline and placed in the other compartment for $1 \mathrm{~h}$. On the day after the final conditioning session, a post-conditioning test that was identical to the pre-conditioning test was performed.

\section{In vivo microdialysis study and quantification of dopamine and its metabolites}

Before implantation of a cannula, all of the rats were anesthetized with sodium pentobarbital $(50 \mathrm{mg} / \mathrm{kg}$, i.p.) for surgery as described previously. Briefly, the anesthetized animal was placed in a stereotaxic apparatus, the skull was exposed, and a small hole was made using a dental drill. A guide cannula (AG-8; Eicom, Kyoto) was implanted into the nucleus accumbens (from the bregma: anterior, $+4.0 \mathrm{~mm}$; lateral, $-0.8 \mathrm{~mm}$; ventral, $-6.8 \mathrm{~mm}$; angle of 16 degrees) according to the atlas of Paxinos and Watson and fixed to the skull with cranioplastic cement. Three to five days after surgery, microdialysis probes (A-I-8-02, 2-mm membrane length; Eicom) were slowly inserted into the nucleus accumbens through guide cannulas under anesthesia with diethyl ether, and the rats were placed in experimental cages $(30 \mathrm{~cm}$ wide $\times 30 \mathrm{~cm}$ deep $\times 30 \mathrm{~cm}$ high). The probes were perfused continuously $(2 \mu \mathrm{l} / \mathrm{min})$ with artificial cerebrospinal fluid: 0.9 $\mathrm{mM} \mathrm{MgCl}_{2}, 147.0 \mathrm{mM} \mathrm{NaCl}, 4.0 \mathrm{mM} \mathrm{KCl}$, and $1.2 \mathrm{mM}$ $\mathrm{CaCl}_{2}$. Outflow fractions were collected every $20 \mathrm{~min}$. After 3 baseline fractions were collected from the rat nucleus accumbens, rats were given morphine $(8 \mathrm{mg} / \mathrm{kg}$, i.p.). Dialysis samples were collected for $180 \mathrm{~min}$ after treatment and analyzed by high-performance liquid chromatography (Eicom) with electrochemical detection
(Eicom). Dopamine (DA) and its metabolites, 3,4dihydroxyphenylacetic acid (DOPAC) and 3-methoxy4-hydroxyphenyl acetic acid (HVA), were separated by column chromatography, and identified and quantified by the use of standards, as described previously (11).

\section{Partial sciatic nerve ligation ( $p S N L$ ) model}

Rats were anesthetized with 3\% isoflurane and partial sciatic nerve injury was produced by tying a tight ligature with 4-0 nylon monofilament (Natsume Seisakusyo) around approximately one half the diameter of the sciatic nerve on the left ipsilateral side, as described previously (23). In the sham operation, the nerve was exposed, but ligation was not performed. Thresholds of $8-15 \mathrm{~g}$ for the contralateral side and $0.6-2 \mathrm{~g}$ for the ipsilateral side at $13-14$ days after the operation were used in the experiments.

\section{Electrical stimulation-induced paw withdrawal (EPW) test}

A small electrode (STE0405; Neurotron, Inc., Baltimore, MD, USA) for stimulation was attached to the right plantar surface of the oxaliplatin and paclitaxel models or the left plantar surface of pSNL models. A skin patch dispersion electrode (SDE44; Neurotron, Inc.) was affixed to the middle of the back of the body, from which the hair had been removed. Rats were kept inside a Ballman cage (Natsume Seisakusyo). Transcutaneous nerve stimuli, specifically 3 sine-wave pulses with frequencies of 2000,250 , or $5 \mathrm{~Hz}$, to activate $\mathrm{A} \beta-, \mathrm{A} \delta$-, or C-fibers, respectively $(24-27)$, were applied to the unilateral hind paw of rats using the animal-response test mode of the Neurometer CPT/C (Neurotron, Inc.). The intensity of each stimulus was gradually increased automatically (increments of $0.05 \mathrm{~mA}$ ). The threshold of current $(\mu \mathrm{A})$ for each stimulus was determined by the vocalization of each rat. The threshold for all three frequencies $(2000,250$, and $5 \mathrm{~Hz})$ in each rat was determined within $2 \mathrm{~min}$, and the procedure was repeated at 10-min intervals. Data represent the means of the values obtained in 2 measurements.

\section{Drugs}

Morphine hydrochloride and oxycodone hydrochloride used in the von Frey test (Fig. 1) were obtained from Shionogi Pharmaceutical (Osaka), and fentanyl citrate used in the von Frey test was obtained from Tyco Healthcare (Tokyo). The drugs used in the CPP test and in vivo microdialysis were morphine hydrochloride (DaiichiSankyo, Tokyo), oxycodone hydrochloride (Shionogi Pharmaceutical Co., Inc., Osaka), and fentanyl citrate (Hisamitsu Pharmaceutical Co., Inc., Tokyo). Paclitaxel and oxaliplatin were purchased from Wako Pure Chemical 
(A)

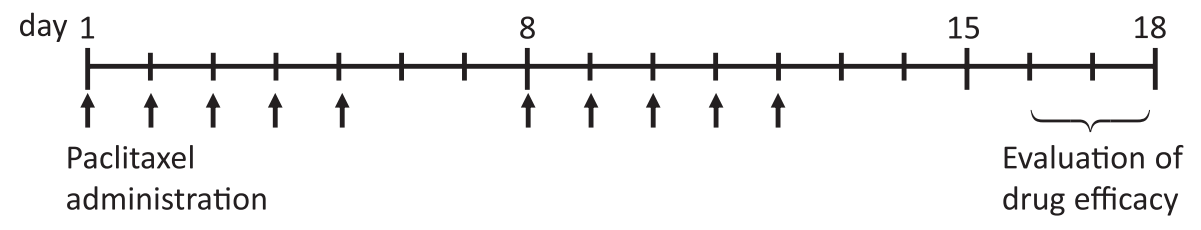

(B)

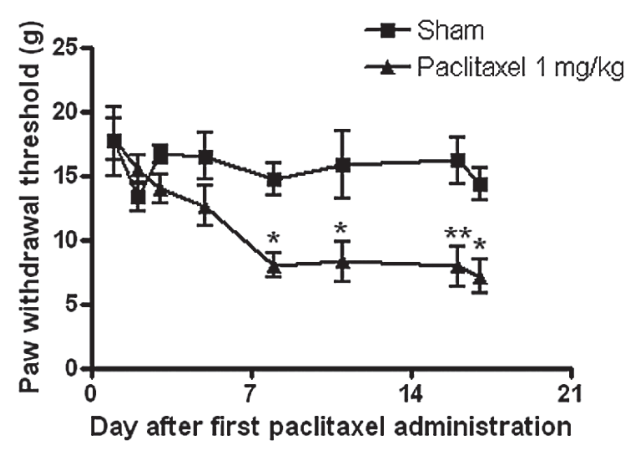

(C)

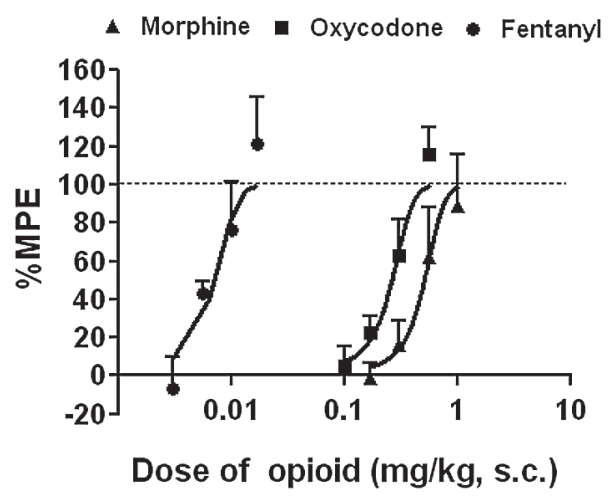

Fig. 1. Expression of mechanical allodynia and the antinociceptive effects of $\mu$-opioid receptor agonists in a paclitaxel model. A) Drug administration schedule. B) The von Frey hair test was performed before the first administration of paclitaxel on day 1 and on days $2,3,5,8,11,16$, and 17. The y-axis represents the paw-withdrawal threshold, expressed as the sum of of the thresholds of the right and left paws (g). ${ }^{*} P<0.05,{ }^{*} P<0.01$ vs. vehicle group (two-way ANOVA, Bonferroni multiple comparison post hoc test). C) Dose-response curves of opioid-induced antinociception in the paclitaxel model based on the von Frey hair test. The antinociceptive effects were calculated as \% MPE. The reported data are each the mean \pm S.E.M. of 5 - 13 rats.

Industries, Ltd. (Osaka). All drugs were dissolved in saline and administered in a volume of $1.0 \mathrm{ml} / \mathrm{kg}$ (rats), except for oxaliplatin and paclitaxel. Oxaliplatin was dissolved in 5\% glucose solution (Otsuka Pharmaceutical, Tokyo), whereas paclitaxel was dissolved in saline including 5\% ethanol and 5\% Cremophore $\mathrm{EL}^{\circledR}$.

\section{Statistical analyses}

Data are expressed as the mean with S.E.M. The statistical significance of differences between groups was assessed by one-way and two-way ANOVA followed by the Bonferroni multiple comparisons test, the Tukey multiple comparisons test, or the Mann-Whitney test (unpaired, two-tailed). The statistical significance of differences between two groups was evaluated by Student's $t$-test. Statistical analyses for data in the EPW test and the place conditioning test were performed using SAS software (ver. 8; SAS Institute, Cary, NC, USA), and other analyses were performed using Prism software (version 5.0a, GraphPad Software). A $P$-value of $<0.05$ was considered to reflect significance.

\section{Results}

Antinociceptive effects of opioids on paclitaxel-induced allodynia in rats

While we previously demonstrated the antinociceptive effects of opioids on oxaliplatin-induced neuropathy in rats (submitted manuscript), there has been no report regarding the effectiveness of opioids on paclitaxelinduced neuropathy in animals. Therefore, we investigated the antinociceptive effects of opioids on paclitaxelinduced neuropathic pain in rats using the von Frey hair test. Repeated treatment with paclitaxel $(1 \mathrm{mg} / \mathrm{kg}$, i.p.; Fig. 1A) induced a significant decrease in the paw-withdrawal threshold from 8 days after the first administration (Fig. 1B). The antinociceptive effects of opioids on paclitaxel-induced allodynia were measured $16-18$ days after the first paclitaxel treatment. Maximal antinociceptive effects were observed $15 \mathrm{~min}$ after s.c. administration of oxycodone $(0.1-0.56 \mathrm{mg} / \mathrm{kg})$ and fentanyl $(0.003-0.017 \mathrm{mg} / \mathrm{kg})$ or $30 \mathrm{~min}$ after the administration of morphine $(0.17-1 \mathrm{mg} / \mathrm{kg}$ ) (data not shown), and these time points were used in doseresponse analyses. Figure $1 \mathrm{C}$ shows the relative effica- 
(A) Morphine

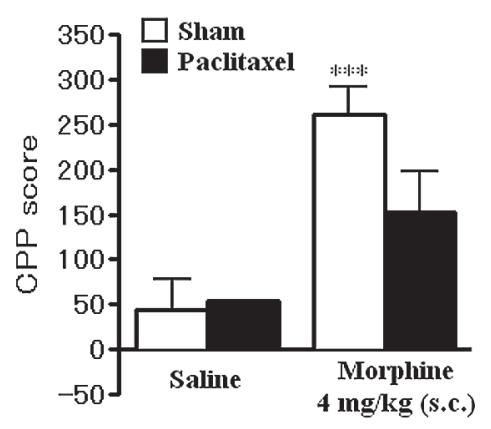

(B) Oxycodone

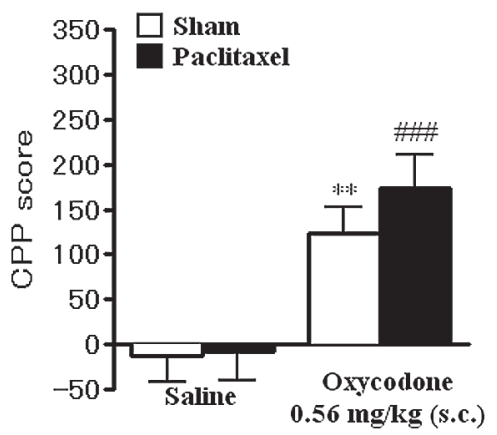

(C) Fentanyl

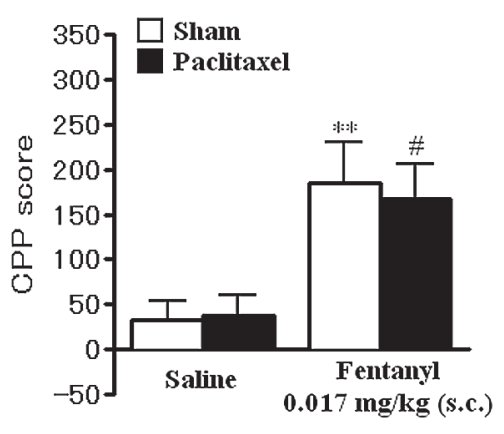

(D) Morphine

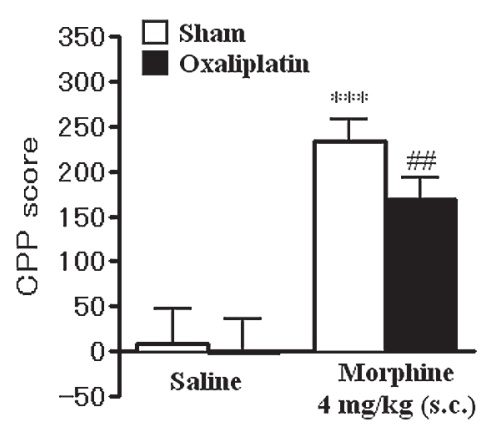

(E) Oxycodone

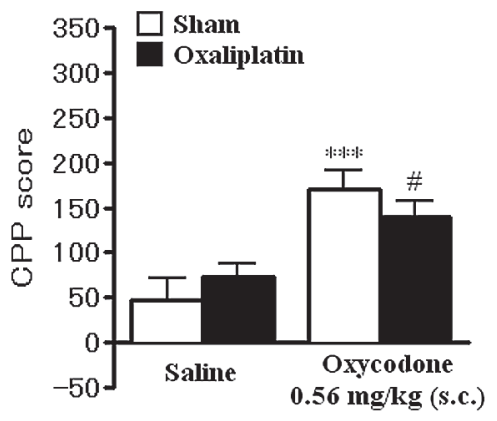

(F) Fentanyl

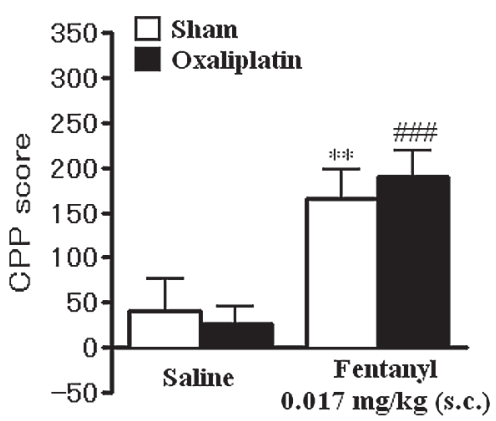

Fig. 2. Effects of paclitaxel- and oxaliplatin-induced neuropathy on the $\mu$-opioid receptor agonist-induced place-preference in rats. Ordinate: mean difference between the time spent in the preferred place in the post-conditioning test and the pre-conditioning test. The reported data are each the mean \pm S.E.M. of $7-8$ rats. ${ }^{* *} P<0.01,{ }^{* * *} P<0.001$ vs. sham-saline groups. ${ }^{\#} P<0.05$, ${ }^{\#} P<0.01,{ }^{\# \#} P<0.001$ vs. paclitaxel-saline or oxaliplatin-saline group (Two-way ANOVA, Tukey multiple comparison post hoc test).

cies of opioids on paclitaxel-induced neuropathy in rats, expressed as the \% MPE for antinociception. All three opioids dose-dependently reversed paclitaxel-induced mechanical neuropathic pain (Fig. 1C), indicating that these opioids had antinociceptive effects against paclitaxel-induced neuropathic pain. In this study, no sedation was observed within the following dose ranges for each opioid: morphine $(0.17-1 \mathrm{mg} / \mathrm{kg}$, s.c. $)$, oxycodone $(0.1-0.56 \mathrm{mg} / \mathrm{kg}$, s.c. $)$, and fentanyl $(0.003-0.017$ $\mathrm{mg} / \mathrm{kg}$, s.c.).

\section{Rewarding effects of opioids on paclitaxel-and oxaliplatin-} induced neuropathy in rats

Previous studies as well as the present study have shown that morphine $(4.0 \mathrm{mg} / \mathrm{kg}$, s.c. $)$, oxycodone $(0.56 \mathrm{mg} / \mathrm{kg}$, s.c. $)$, and fentanyl $(0.017 \mathrm{mg} / \mathrm{kg})$ produce a significant place preference in naïve rats $(12,28,29)$.
In the present study, morphine-, oxycodone-, and fentanylinduced place preferences were marginally affected by paclitaxel-induced neuropathy (Fig. 2: A-C). These results surprised us a bit, since other studies have shown that the rewarding effects induced by morphine could be suppressed under an inflammatory (carrageenan- and formalin-induced pain) $(11,13)$ and a neuropathic pain (partial sciatic nerve ligation-induced pain)-like state (12) in rodents. In this study, it is possible that the relief from paclitaxel-induced neuropathy seen with $\mu$-opioid agonists may be closely related to induction of the place preference by $\mu$-opioid agonists without the induction of rewarding effects. Thus, the place aversion induced by paclitaxel-induced neuropathic pain may reflect a false-positive place preference induced by the antinociceptive effects of $\mu$-opioid agonists. In fact, $\mu$-opioid agonists could potently suppress paclitaxel-induced 


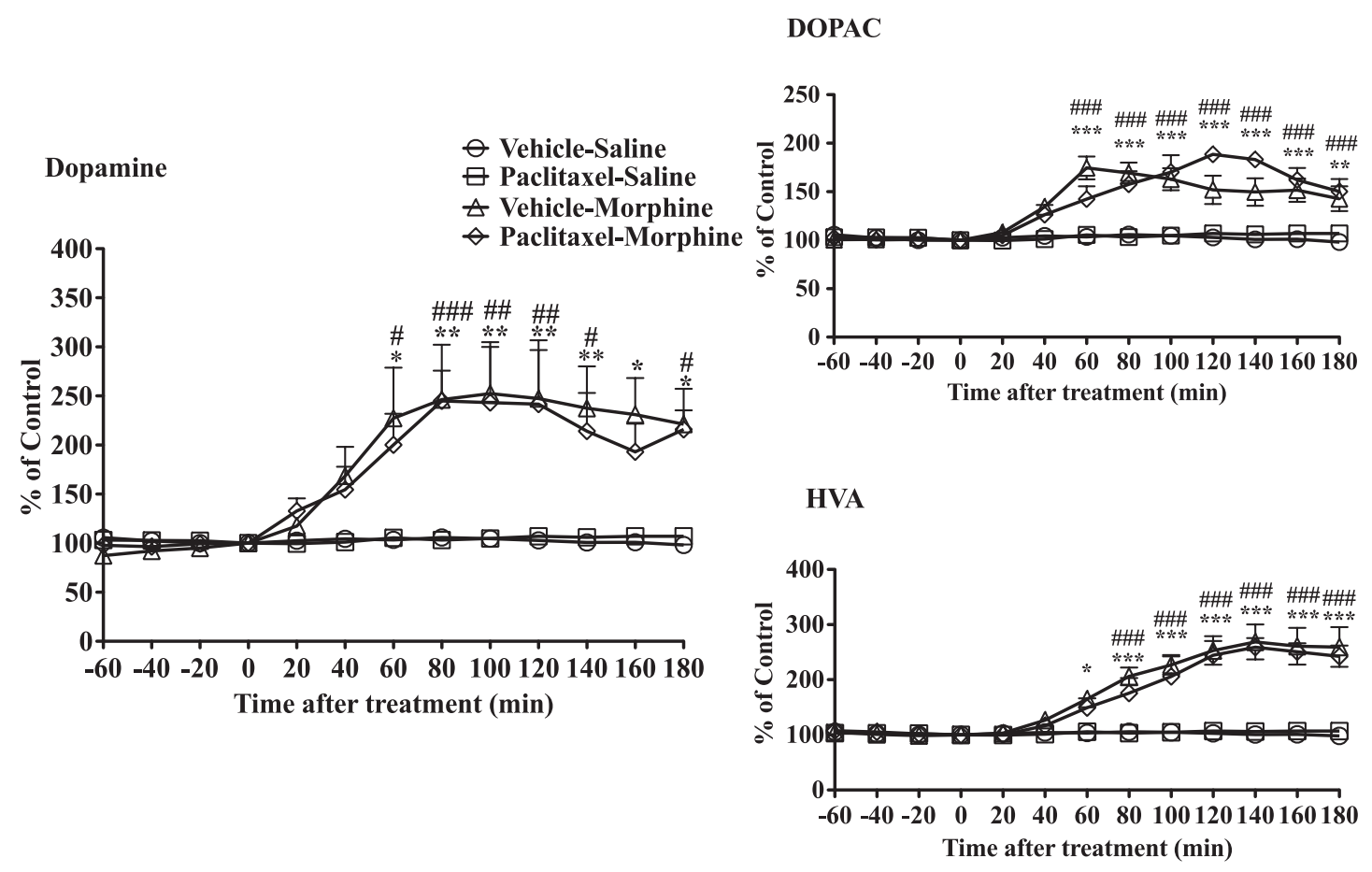

Fig. 3. Effects of paclitaxel-induced neuropathy on the influence of morphine on the dialysate concentrations of dopamine in the nucleus accumbens. After baseline fractions were collected, rats were injected with morphine $(8 \mathrm{mg} / \mathrm{kg})$ at time 0 to evoke the release of dopamine. Data are expressed as percentages of the corresponding baseline levels with S.E.M. for 6 rats under the influence of morphine on the dialysate concentrations of dopamine and its metabolites in the nucleus accumbens. Statistical analyses were performed with two-way ANOVA followed by the Bonferroni multiple comparisons test: vehicle-saline vs. vehiclemorphine, $\mathrm{F}(1,117)=49.41, P<0.0001$; Saline-morphine vs. Paclitaxel-morphine, $\mathrm{F}(1,150)=0.58, P>0.05$.

neuropathic pain (Fig. 1C). To explore this possibility, we next examined the effects of oxaliplatin-induced neuropathy on the rewarding effects of $\mu$-opioid agonists, since morphine and oxycodone sufficiently relieved oxaliplatin-induced neuropathy, while fentanyl did not produce antinociception against oxaliplatin-induced neuropathic pain (submitted manuscript). As a result, morphine- and oxycodone-induced rewarding effects were not suppressed under oxaliplatin-induced neuropathy (Fig. 2: D and E). Importantly, we also found that fentanyl-induced rewarding effects were established under oxaliplatin-induced neuropathy regardless of this insufficient antinociception (Fig. 2F), suggesting that chemotherapeutic drug-induced neuropathy may not suppress the development of $\mu$-opioid receptor agonistinduced rewarding effects.

Increase in the release of dopamine from the nucleus accumbens by morphine in the paclitaxel model

We previously reported that the increase in the release of dopamine from the NAc by morphine was suppressed under inflammatory pain and pSNL-induced pain conditions, which is closely related to the abolishment of the rewarding effects of morphine $(11,12)$. Therefore, we evaluated morphine-induced dopamine release from the NAc in rats that were suffering from paclitaxel-induced neuropathy using in vivo microdialysis. In this study, we selected $8 \mathrm{mg} / \mathrm{kg}$ of morphine, since this dose of morphine can stably increase the release of dopamine from the NAc. The basal extracellular levels of DA, DOPAC, and HVA in the NAc of control rats were $5.14 \pm 1.68 \mathrm{nM}, 4.904 \pm 0.951 \mu \mathrm{M}$, and $2.62 \pm 0.460$ $\mu \mathrm{M} / 20$ min (mean with S.E.M. of 5 samples), respectively. The basal extracellular levels of DA, DOPAC, and HVA in the NAc of paclitaxel-treated rats were $10.43 \pm 2.285 \mathrm{nM}, 9.413 \pm 1.235 \mu \mathrm{M}$, and $4.45 \pm 0.724$ $\mu \mathrm{M} / 20$ min (mean with S.E.M. of 5 samples), respectively. The levels of dopamine and its metabolites in paclitaxel-treated rats were higher than those in control rats, but these changes were not significant. Morphine significantly increased the release of dopamine from the NAc, as well as the DOPAC and HVA levels, in both sham and paclitaxel-treated rats; and the morphineinduced increase in the release of dopamine from the NAc was not altered under paclitaxel-induced neuropathy (Fig. 3). These results indicate that activation 
of the mesolimbic dopaminergic system induced by morphine was hardly affected by paclitaxel-induced neuropathy. This phenomenon supports the idea that $\mu$ opioid receptor agonist-induced rewarding effects are present even under paclitaxel-induced neuropathic pain.

Qualitative difference between chemotherapeutic-induced neuropathy models and a partial sciatic nerve ligation (pSNL) model

As in the pSNL model, chemotherapeutic drugs (oxaliplatin and paclitaxel) produced allodynia-like behaviors using the von Frey test (Fig. 1B). However, the clinical symptoms in the two types of neuropathy seem to be different $(18-20)$. Therefore, we hypothesized that pSNL and chemotherapeutic drugs induce neuropathic pain through different underlying mechanisms, and in turn these distinct pain mechanisms may differentially regulate the activation of the mesolimbic dopaminergic system induced by morphine. In this study, we performed an EPW test to confirm that there was a qualitative difference between the neuropathies induced by pSNL and chemotherapeutic drugs. In the EPW test, a dramatic difference was observed in $\mathrm{A} \beta$ fibers. The threshold for $2000 \mathrm{~Hz}$ (A $\beta$ fiber) was significantly reduced in paclitaxel- and oxaliplatin-treated rats, but not in the pSNL model (Fig. 4). The threshold for $250 \mathrm{~Hz}$ (A $\delta$ fiber) was slightly (but not significantly) decreased in all models (Fig. 4). Furthermore, the threshold for $5 \mathrm{~Hz}$ (C fiber) tended to be reduced in the paclitaxel and pSNL models (Fig. 4: A and C). These results suggest that the different activation patterns for pain-related fibers may explain why chemotherapeutic drug-induced neuropathy did not suppress the establishment of the rewarding effects of $\mu$-opioid receptor agonists, particularly morphine.

\section{Discussion}

The present study showed that chemotherapeutic drug (oxaliplatin and paclitaxel)-related neuropathy in rats does not affect the establishment of the rewarding effects of $\mu$-opioid receptor agonists. We have shown that morphine-induced rewarding effects could be suppressed under an inflammatory (carrageenan- and formalin-induced pain) (13) or a neuropathic pain (partial sciatic nerve ligation-induced pain)-like state (12), which is accompanied by suppression of the activation of the mesolimbic dopaminergic system induced by morphine $(11,12,14)$. It should be noted here that inflammatory pain upregulates the function of the dynorphinergic system in the NAc (11), whereas neuropathic pain downregulates $\mu$-opioid receptor function in the VTA (14) to negatively regulate the activation of the mesolimbic
(A) Paclitaxel model

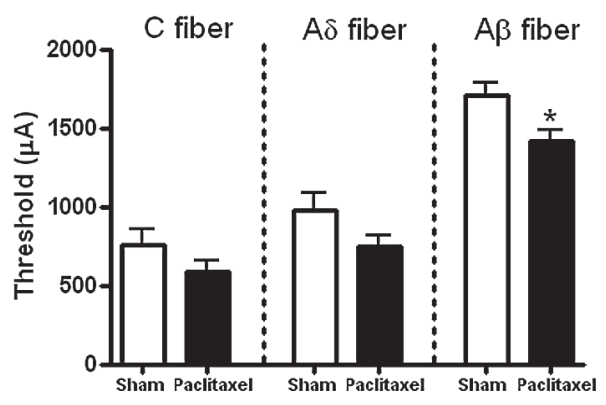

(B) Oxaliplatin model

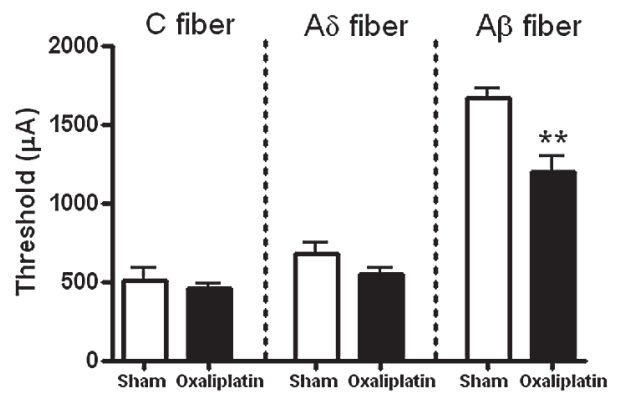

(C) pSNL model

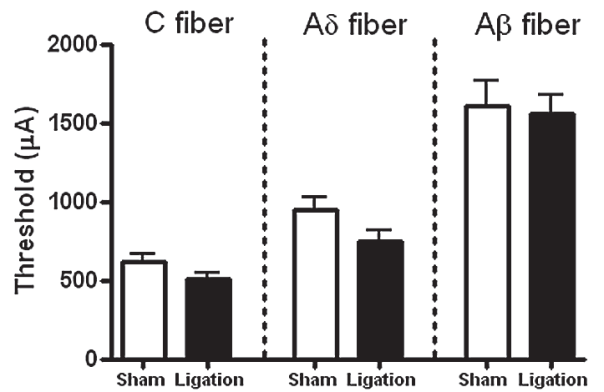

Fig. 4. Thresholds of $\mathrm{A} \beta, \mathrm{A} \delta$ and $\mathrm{C}$ fibers in the oxaliplatin, paclitaxel and pSNL models. Thresholds for pain by the activation of $\mathrm{A} \beta, \mathrm{A} \delta$, and $\mathrm{C}$ fibers in the paclitaxel (A), oxaliplatin (B), and pSNL (C) models. The threshold represents the minimum intensity $(\mu \mathrm{A})$ required for vocalization in response to electrical stimulation for each fiber. Data represent the mean \pm S.E.M. of $6-13$ rats. ${ }^{*} P<0.05$, $* * P<0.01$ vs. sham group for each fiber (Student's $t$-test).

dopaminergic system by morphine as neuroplastic responses. In the present study, the activation of the mesolimbic dopaminergic system produced by morphine was not altered under paclitaxel-induced neuropathy. Therefore, the mechanism of action of chemotherapeutic drug-induced neuropathy could not neuroplastically affect the activation of the mesolimbic dopaminergic system produced by $\mu$-opioid receptor agonists. Therefore, the rewarding effects of $\mu$-opioid receptor agonists can be established under oxaliplatin- and paclitaxelinduced pain. 
Although $\mu$-opioid receptor agonists have antinociceptive effects against chemotherapeutic drug-induced neuropathic pain, fentanyl seems to possess a different antinociceptive potency, which depends on the chemotherapeutic drug. In particular, fentanyl produced sufficient antinociception in paclitaxel-induced neuropathic pain, while the antinociceptive effects of fentanyl were restricted against oxaliplatin-induced neuropathic pain in our previous study (submitted manuscript). We also showed that the very limited antinociception produced by fentanyl results from a loss of fentanyl-sensitive Gi/o protein activation for neuropathic pain induced by oxaliplatin (submitted manuscript). Therefore, oxaliplatin may alter the fentanyl-modulated pain-related signal transduction at $\mu$-opioid receptor and/or G-protein regulation to induce antinociceptive effects. We interestingly demonstrated that fentanyl-induced rewarding effect could be established even in the presence of oxaliplatin-induced neuropathic pain. These findings suggest that opioid-induced psychological dependence may develop even though pain is still present under certain conditions.

Slight neuronal changes in $\mathrm{A} \delta$ and $\mathrm{C}$ fibers were observed in both pSNL- and chemotherapeutic druginduced neuropathic pain. These changes might reflect the pain symptoms, such as hyperalgesia and allodynia, seen under neuropathic pain. In addition, the threshold of A $\beta$ fibers was significantly changed in chemotherapyinduced neuropathic pain, but not in pSNL-induced pain. It has been demonstrated that patients who are treated with chemotherapeutic drugs exhibit not only pain, such as hyperalgesia and allodynia, but also tactile symptoms, such as numbness and paresthesia (15). Therefore, the neuronal change in $\mathrm{A} \beta$ fibers in chemotherapy-induced neuropathy may reflect tactile symptoms. Thus, distinct patterns of the modulation of pain-related fibers induced by chemotherapeutic drugs or pSNL would lead to several phenotypes of neuropathic-like pain $(18-20)$. On the other hand, a previous study showed that the application of morphine to the spinal cord attenuated $\mathrm{C}$ fiber-, but not $\mathrm{A} \beta$ fiber-, evoked spinal neuron responses after spinal nerve ligation (30). Therefore, it is possible that $\mathrm{A} \beta$ fiber-related pain is relatively opioid-resistant.

In conclusion, control of chemotherapeutic druginduced neuropathic pain is a serious problem in any anti-cancer program, since limited methodologies have been established to treat neuropathy. Our previous and present studies have suggested that $\mu$-opioid receptor agonists can effectively reduce the neuropathy induced by chemotherapeutic drugs. However, the present study demonstrated that the rewarding effects of $\mu$-opioid receptor agonists were not suppressed under chemotherapeutic drug-induced neuropathy. Therefore, the reward- ing/reinforcing effects of $\mu$-opioid receptor agonists should be carefully monitored when they are used to control chemotherapeutic drug-induced allodynia. It is still unclear why oxaliplatin-induced neuropathic pain is resistant to the antinociceptive effects of fentanyl and why the rewarding effects of $\mu$-opioid receptor agonists could still be established under chemotherapeutic druginduced neuropathic pain. Therefore, further studies will be needed to explore these issues.

\section{Acknowledgments}

This work was supported in part by Ministry of Education, Culture, Sports, Science and Technology of Japan and also supported by Shionogi \& Co., Ltd.

\section{References}

1 Woodcock J. A difficult balance--pain management, drug safety, and the FDA. N Engl J Med. 2009;361:2105-2107.

2 Liu SB, Ma L, Guo HJ, Feng B, Guo YY, Li XQ, et al. Gentiopicroside attenuates morphine rewarding effect through downregulation of GluN2B receptors in nucleus accumbens. CNS Neurosci Ther. 2012;18:652-658.

3 Popik P, Mamczarz J, Fraczek M, Widla M, Hesselink M, Danysz W. Inhibition of reinforcing effects of morphine and naloxone-precipitated opioid withdrawal by novel glycine site and uncompetitive NMDA receptor antagonists. Neuropharmacology. 1998;37:1033-1042.

4 Valjent E, Corbille AG, Bertran-Gonzalez J, Herve D, Girault JA. Inhibition of ERK pathway or protein synthesis during reexposure to drugs of abuse erases previously learned place preference. Proc Natl Acad Sci U S A. 2006;103:2932-2937.

5 Benturquia N, Le Marec T, Scherrmann JM, Noble F. Effects of nitrous oxide on dopamine release in the rat nucleus accumbens and expectation of reward. Neuroscience. 2008;155: 341-344.

6 Koob GF. Neural mechanisms of drug reinforcement. Ann N Y Acad Sci. 1992;654:171-191.

7 Nestler EJ. Under siege: the brain on opiates. Neuron. 1996; 16:897-900.

8 Pothos E, Rada P, Mark GP, Hoebel BG. Dopamine microdialysis in the nucleus accumbens during acute and chronic morphine, naloxone-precipitated withdrawal and clonidine treatment. Brain Res. 1991;566:348-350.

9 Eisenberg E, McNicol ED, Carr DB. Efficacy and safety of opioid agonists in the treatment of neuropathic pain of nonmalignant origin: systematic review and meta-analysis of randomized controlled trials. JAMA. 2005;293:3043-3052.

10 Passik SD. Issues in long-term opioid therapy: unmet needs, risks, and solutions. Mayo Clin Proc. 2009;84:593-601.

11 Narita M, Kishimoto Y, Ise Y, Yajima Y, Misawa K, Suzuki T. Direct evidence for the involvement of the mesolimbic kappaopioid system in the morphine-induced rewarding effect under an inflammatory pain-like state. Neuropsychopharmacology. 2005;30:111-118.

12 Ozaki S, Narita M, Iino M, Sugita J, Matsumura Y, Suzuki T. Suppression of the morphine-induced rewarding effect in the 
rat with neuropathic pain: implication of the reduction in mu-opioid receptor functions in the ventral tegmental area. J Neurochem. 2002;82:1192-1198.

13 Suzuki T, Kishimoto Y, Misawa M. Formalin- and carrageenaninduced inflammation attenuates place preferences produced by morphine, methamphetamine and cocaine. Life Sci. 1996;59: 1667-1674.

14 Niikura K, Narita M, Butelman ER, Kreek MJ, Suzuki T. Neuropathic and chronic pain stimuli downregulate central mu-opioid and dopaminergic transmission. Trends Pharmacol Sci. 2010;31:299-305.

15 Argyriou AA, Cavaletti G, Briani C, Velasco R, Bruna J, Campagnolo $\mathrm{M}$, et al. Clinical pattern and associations of oxaliplatin acute neurotoxicity: a prospective study in 170 patients with colorectal cancer. Cancer. 2013;119:438-444.

16 Inoue K, Nagai S, Kaneko S, Uchida S, Hayashi Y, Higuchi $\mathrm{T}$, et al. [Efficacy of oxycodone against anti-cancer agent-induced pain in breast cancer patients during adjuvant treatment before and after surgery]. Gan To Kagaku Ryoho. 2011;38:1137-1142. (text in Japanese with English abstract)

17 Nagashima M, Ooshiro M, Moriyama A, Sugishita Y, Kadoya $\mathrm{K}$, Sato A, et al. Efficacy and tolerability of controlled-release oxycodone for oxaliplatin-induced peripheral neuropathy and the extension of FOLFOX therapy in advanced colorectal cancer patients. Support Care Cancer. 2014;22:1579-1584.

18 Baron R, Tolle TR, Gockel U, Brosz M, Freynhagen R. A crosssectional cohort survey in 2100 patients with painful diabetic neuropathy and postherpetic neuralgia: differences in demographic data and sensory symptoms. Pain. 2009;146:34-40.

19 Kautio AL, Haanpaa M, Saarto T, Kalso E. Amitriptyline in the treatment of chemotherapy-induced neuropathic symptoms. J Pain Symptom Manage. 2008;35:31-39.

20 Wolf S, Barton D, Kottschade L, Grothey A, Loprinzi C. Chemotherapy-induced peripheral neuropathy: prevention and treatment strategies. Eur J Cancer. 2008;44:1507-1515.

21 Dina OA, Chen X, Reichling D, Levine JD. Role of protein kinase Cepsilon and protein kinase A in a model of paclitaxelinduced painful peripheral neuropathy in the rat. Neuroscience. 2001;108:507-515.

22 Suzuki T, Funada M, Narita M, Misawa M, Nagase H. Pertussis toxin abolishes mu- and delta-opioid agonist-induced place preference. Eur J Pharmacol. 1991;205:85-88.

23 Seltzer Z, Dubner R, Shir Y. A novel behavioral model of neuropathic pain disorders produced in rats by partial sciatic nerve injury. Pain. 1990;43:205-218.

24 Liu S, Kopacz DJ, Carpenter RL. Quantitative assessment of differential sensory nerve block after lidocaine spinal anesthesia. Anesthesiology. 1995;82:60-63.

25 Masson EA, Boulton AJ. The Neurometer: validation and comparison with conventional tests for diabetic neuropathy. Diabet Med. 1991;8:S63-S66.

26 Pitei DL, Watkins PJ, Stevens MJ, Edmonds ME. The value of the Neurometer in assessing diabetic neuropathy by measurement of the current perception threshold. Diabet Med. 1994; 11:872-876.

27 Tay B, Wallace MS, Irving G. Quantitative assessment of differential sensory blockade after lumbar epidural lidocaine. Anesth Analg. 1997;84:1071-1075.

28 Liu YL, Yan LD, Zhou PL, Wu CF, Gong ZH. Levo-tetrahydropalmatine attenuates oxycodone-induced conditioned place preference in rats. Eur J Pharmacol. 2009;602:321-327.

29 Miller DK, Nation JR. Chronic cadmium exposure attenuates the conditioned reinforcing properties of morphine and fentanyl. Brain Res. 1997;776:162-169.

30 Suzuki R, Chapman V, Dickenson AH. The effectiveness of spinal and systemic morphine on rat dorsal horn neuronal responses in the spinal nerve ligation model of neuropathic pain. Pain. 1999;80:215-228. 